\title{
Photochemical conversion of tin-oxo cage compounds studied using hard x-ray photoelectron spectroscopy
}

\author{
Yu Zhang ${ }^{\mathrm{a}}$, Jarich Haitjema ${ }^{\mathrm{a}}$, Xiaomeng Liu ${ }^{\mathrm{a}}$, Fredrik Johansson, ${ }^{\mathrm{b}}$ Andreas Lindblad, ${ }^{\mathrm{b}}$ Sonia \\ Castellanos $^{\mathrm{a}}$, Niklas Ottosson ${ }^{\mathrm{a}, \mathrm{c}}$, Albert M. Brouwer ${ }^{\mathrm{a}, \mathrm{d}}$ \\ ${ }^{a}$ Advanced Research Center for Nanolithography, P.O. Box 93019, 1090 BA Amsterdam, The \\ Netherlands \\ ${ }^{b}$ Dept. Physics and Astronomy, Div. Mol \& Cond. Mat. Phys., Uppsala University, Box 516, SE- \\ 75120, Uppsala, Sweden \\ 'AMOLF, Science Park 104, 1098 XG Amsterdam, The Netherlands \\ dvan 't Hoff Institute for Molecular Sciences, Faculty of Science, University of Amsterdam, P.O. \\ Box 94157, 1090 GD Amsterdam, The Netherlands
}

\begin{abstract}
Several metal-containing molecular inorganic materials are currently considered as photoresists for extreme ultraviolet lithography (EUVL). This is primarily due to their high EUV absorption cross section and small building block size, properties which potentially allow both high sensitivity and resolution as well as low line-edge roughness. The photochemical reaction mechanisms that allow these kinds of materials to function as photoresists, however, are still poorly understood. As a step in this direction, we here discuss photochemical reactions upon deep UV (DUV) irradiation of a model negative-tone EUV photoresist material, namely the well-defined molecular tin-oxo cage compound $\left[(\mathrm{SnR})_{12} \mathrm{O}_{14}(\mathrm{OH})_{6}\right] \mathrm{X}_{2}(\mathrm{R}=$ organic group; $\mathrm{X}=$ anion $)$ which is spin coated to thin layers of $20 \mathrm{~nm}$. The core electronic structure (Sn 3d, O 1s and C 1s) of fresh and DUV exposed films were then investigated using synchrotron radiationbased hard X-ray photoelectron spectroscopy (HAXPES). This method provides information about the structure and chemical state of the respective atoms in the material. We performed a comparative HAXPES study of the composition of the tin-oxo cage compound $\left[(\mathrm{SnR})_{12} \mathrm{O}_{14}(\mathrm{OH})_{6}\right](\mathrm{OH})_{2}$, either fresh directly after spin-coated vs. DUV-exposed materials under either ambient condition or under a dry $\mathrm{N}_{2}$ atmosphere. Different chemical oxidation states and concentrations of atoms and atom types in the fresh and exposed films were found. We further found that the chemistry resulting from exposure in air and $\mathrm{N}_{2}$ is strikingly different, clearly illustrating the influence of film-gas interactions on the (photo)chemical processes that eventually determine the photoresist. Finally, a mechanistic hypothesis for the basic DUV photoreactions in molecular tin-oxo cages is proposed.
\end{abstract}

Keywords: Inorganic photoresist, tin oxo cages, HAXPES, photochemical mechanism, DUV

\section{INTRODUCTION}

Metal-containing hybrid inorganic materials are considered promising candidates as photoresists in high volume semiconductor manufacturing using extreme ultraviolet lithography (EUVL), ever since these materials were introduced in the past years [1-5]. Photoresists based on $\mathrm{HfO}_{2}$ and $\mathrm{ZrO}_{2}$ nanoparticles were synthesized and shown to yield pattern formation under EUVL by Ober's group.[3] Tin-oxo cages[6], palladium complexes[7], organotin compounds[8] and other molecular compounds have further been explored as EUV photoresist by Brainard's group. Recently, Inpria introduced Sn-based hybrid photoresists.[5] Compared to traditional organic chemically amplified photoresists (CARs), some of these inorganic photoresists show higher sensitivity, can form thinner layers and yield both lower line edge roughness and higher resolution. Such findings have shown the great potential for inorganic materials to be used as EUV photoresist. CARs have now been used and optimized for many years, first for DUV and more recently for EUV, and their critical photochemical reactions are generally well understood.[9] The limitation of CARs in EUVL, however, have been clearly identified.[10] In contrast, the photoreaction mechanisms in the inorganic photoresist have not been

Advances in Patterning Materials and Processes XXXIV, edited by Christoph K. Hohle, Proc. of SPIE Vol. 10146, $1014606 \cdot$ • (c) 2017 SPIE · CCC code: 0277-786X/17/\$18 · doi: 10.1117/12.2257893 
thoroughly investigated yet. In order to further improve the performance of the inorganic photoresists, systematic studies of the key photoreaction mechanisms inside the photoresist are necessary.

In this contribution we study the structure and photochemistry in spin-coated layers of the tin-oxo cage compound $\left[(\mathrm{SnR})_{12} \mathrm{O}_{14}(\mathrm{OH})_{6}\right](\mathrm{OH})_{2}$, which can be seen as an ideal model system of inorganic nano-scale photoresists, given its well-defined metal-oxide core-structure with surrounding n-butyl organic ligands and hydroxide counterions; see Fig. 1. In order to synthesize the compound we have followed the route in reference [12]. The molecule contains $\mathrm{Sn}$ atoms with high EUV photon absorption, $[13,14]$ and it has been demonstrated to work as an EUV photoresist $[6,8]$. Despite the relatively simple structure of this molecular system, few spectroscopic methods are suitable to study the details of the photochemical reactions occurring in such thin films, both due to limited sensitivity and chemical contrast. Here we apply one of the ideal techniques that fulfil these demands, namely hard X-ray photoelectron spectroscopy (HAXPES) with tunable high photon energies from synchrotron sources, typically ranging from $1.7 \mathrm{keV}$ to $10 \mathrm{keV}$. The basis of the technique is photoionization from a large range of valence and core levels. While core-level photoemission naturally gives rise to elemental sensitivity (e.g. carbon and oxygen emit electrons from core levels in completely separate and well-defined energy ranges) the fine chemical shifts in the binding energies of inequivalent atomic sites makes the method highly chemically sensitive[15]. Finally, owing to the high kinetic energy of the outgoing photo-electrons achievable for low binding energy core and valence levels with high photon energy molecular layers far below the surface can be probed, since the electrons' inelastic mean free path increase with their kinetic energy. Thus, the bulk properties of the spin-coated photoresist layer can be accessed, before and after exposure.[11]

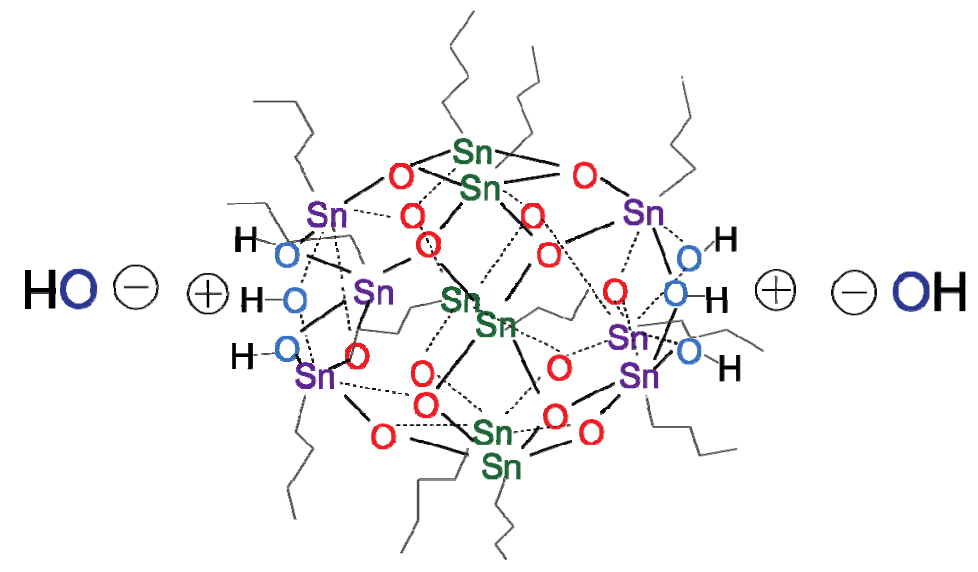

Figure 1) Molecular structure of the $\left[(\mathrm{SnBu})_{12} \mathrm{O}_{14}(\mathrm{OH})_{6}\right](\mathrm{OH})_{2}$ tin-oxo cage compound.

\section{MATERIALS AND METHODS}

\subsection{Materials}

The tin oxo cage with tosylates as counterions (TinS) was synthesized following the procedure in reference[12], starting from butylstannoic acid hydrate and p-toluene sulfonic acid monohydrate [12]. Tin-oxo cages with hydroxide as counterions ( $\mathrm{TinOH})$ were obtained by mixing a solution of aqueous tetramethylammonium hydroxide (TMAH) with a solution of TinS in isopropanol. TinOH precipitated and the product was filtered directly. Results of a more extensive series of tin cage compounds will be published elsewhere. Glass coverslip were purchased from Menzel Gläser and cut into 1 by $1 \mathrm{~cm}^{2}$ squares.

\subsection{Photoresists film preparation}

For photoelectron spectroscopy experiments it is essential that the substrates are conducting. These were prepared by coating piranha base-cleaned glass coverslips with $2 \mathrm{~nm} \mathrm{Cr}$ and $18 \mathrm{~nm}$ Au layers using a sputter coater (Leica EM ACE 
600 Double sputter coater). The tin oxo-cage material was dissolved in toluene to a concentration of $7.5 \mathrm{mg} / \mathrm{mL}$. Solutions were filtered through a $0.25 \mu \mathrm{m}$ PTFE filter right before spin coating. Thin layers $(\sim 20 \mathrm{~nm}$, determined by AFM) were obtained by spin-coating under $2000 \mathrm{rpm}$ for $45 \mathrm{~s}$ with a speed of $750 \mathrm{rpm} / \mathrm{s}$.

\subsection{Photoresists exposure}

A YAG-pumped OPA laser (Ekspla NT342B), delivering nanosecond pulses at $225 \mathrm{~nm}$ at $10 \mathrm{~Hz}(2.5 \mathrm{~mJ} / \mathrm{pulse}) \mathrm{was}$ used as the irradiation source. Some samples were exposed under ambient conditions with a dose of $1.3 \mathrm{~J} / \mathrm{cm}^{2}$. The other samples were exposed in an enclosed sample chamber, containing dry $\mathrm{N}_{2}$, to the same dose (i.e. the exposure time was corrected for the losses due to window reflectivity).

\subsection{Characterization}

All samples were characterized using HAXPES at the HIKE end station, located at the KMC-1 bending-magnet beamline at BESSY-II, Helmholtz-Zentrum-Berlin[16]. The photoelectron spectra were collected with a VG Scienta R4000 hemispherical electron energy analyser which can analyze electron kinetic energies up to $10 \mathrm{keV}$. The pressure in the experimental chamber was in the low $10^{-9}$ mbar range and the samples were introduced via a load-lock chamber. The photon energy was here set to $2005 \mathrm{eV}$ and variation of the photon energy was only done to unambiguously assign features as direct photoionization lines with variable kinetic energy (e.g. in contrast to Auger transitions that have constant kinetic energy). The total energy resolution of the experiment, with the main contributions coming from the photon bandwidth and the electron analyzer resolution, was estimated to approx. $250 \mathrm{meV}$. Two types of spectra were recorded; i) overview spectra taken from $0-800 \mathrm{eV}$ binding energy (BE) in which all relevant lines were included, and ii) detail core-level spectra of the $\mathrm{Sn} 3 \mathrm{~d}, \mathrm{C} 1 \mathrm{~s}$ and $\mathrm{O} 1 \mathrm{~s}$ regions. The former spectra were used to extract atomic number ratios, whereas the latter were used to resolve the chemical shifts in the respective elemental region.

\section{RESULT AND DISCUSSION}

\subsection{Atomic concentration of different samples}

Overview HAXPES spectra of fresh TinOH thin films, as well as samples exposed to DUV in air and in $\mathrm{N}_{2}$ are shown in Fig. 2. All the peaks are marked with the electronic state from which the electrons originate. Au $4 \mathrm{f}$ peaks, originating from the layer underneath the tin cage layer, are present in the spectra and were used for binding energy calibration; the Au $4 \mathrm{f}_{7 / 2}$ binding energy was taken to be $84.00 \mathrm{eV}$.[17] The tin cage gives rise to clear $\mathrm{Sn} 3 \mathrm{~d}$ peaks at $\sim 487$ and $\sim 498 \mathrm{eV}$ (5/2 and 3/2 spin-orbit components, respectively), an $\mathrm{O} 1 \mathrm{~s}$ peak at $\sim 531 \mathrm{eV}$, and a $\mathrm{C} 1 \mathrm{~s}$ peak at $\sim 284 \mathrm{eV}$. The peak positions correspond well with those in the literature. [11, 18, 19,20]

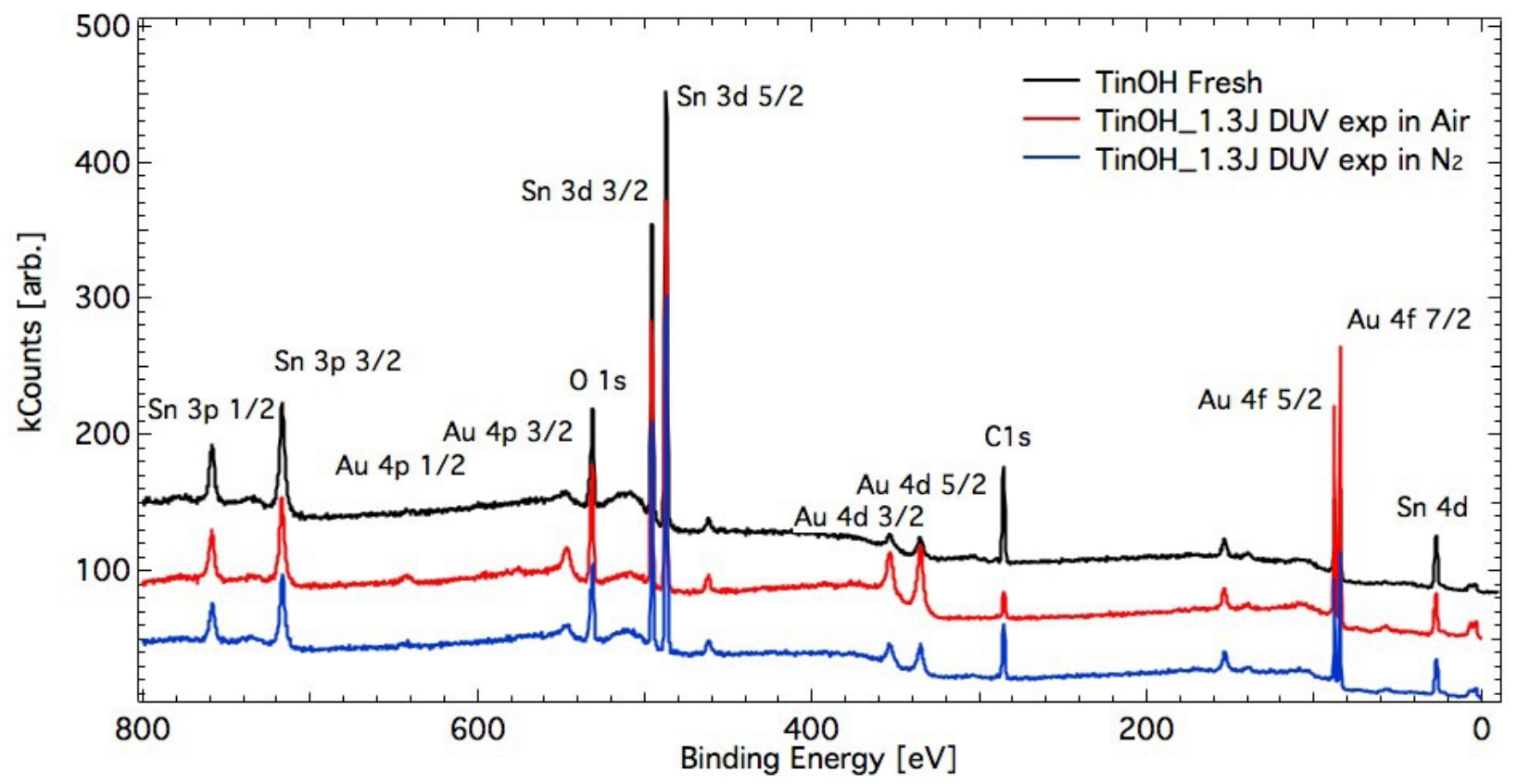

Figure 2) Overview HAXPES spectrum of TinOH fresh sample, TinOH DUV-exposed in air and TinOH DUV-exposed in $\mathrm{N}_{2}$. 
The atomic ratios of $\mathrm{Sn}, \mathrm{O}$ and $\mathrm{C}$ atoms inside the three samples are proportional to the area ratios of the peaks inside the overview spectrum, and can be extracted with knowledge of the photoionization cross sections.[21] For the fresh sample, the extracted atomic ratios correspond well within the error bar to the expected chemical composition. For the exposed samples, the O1s and C1s peaks area are normalized to the Sn peak area, and the atom ratios for these samples are given in Table 1.

Table 1) Atomic ratios of TinOH fresh sample, TinOH DUV-exposed in air and TinOH DUV-exposed in $\mathrm{N}_{2}$.

\begin{tabular}{lccc}
\hline Atom no. & $\mathrm{Sn}$ & $\mathrm{O}$ & $\mathrm{C}$ \\
\hline Expected & 12 & 22 & 48 \\
TinOH_fresh & 12 & $22 \pm 1$ & $48 \pm 1$ \\
DUV exp air & 12 & $32 \pm 1$ & $18 \pm 1$ \\
DUV exp N2 & 12 & $22 \pm 1$ & $36 \pm 1$ \\
\hline
\end{tabular}

As can be seen from Table 1, after the TinOH sample was exposed to DUV in air, the oxygen content was significantly increased while the carbon content was drastically decreased. This suggests that oxidation happens during the exposure, together with $\mathrm{Sn}-\mathrm{C}$ bond or $\mathrm{C}-\mathrm{C}$ bond cleavage, causing a loss of volatile carbon-containing species. When the sample was exposed in dry $\mathrm{N}_{2}$, however, the $\mathrm{O}$ content stayed the same; when no oxygen-containing gas-phase species is present no further oxidation is possible and the photo-induced outgassing apparently does not cause any further loss of oxygen either. Compared to the carbon loss when TinOH was exposed in air, less $\mathrm{C}$ was lost under the same DUV dose.

\subsection{Analysis of $\mathrm{Sn}, \mathrm{O}$ and $\mathrm{C}$ chemical shifts}

The high-resolution $S n 3 d_{5 / 2}$ photoelectron spectra of the respective sample are shown in Fig. 3a. While small, wellreproducible shifts can clearly be observed which we interpret as a result of change of the Sn oxidation state. The dependency of the Sn 3d binding energy on the Sn oxidation state has been well established in the literature.[11] The center of the $\operatorname{Sn} 3 \mathrm{~d}_{5 / 2}$ peak after DUV exposure in air is shifted to higher binding energy, indicating that the oxidation state of the Sn atoms has increased. In contrast, the center energy position after DUV exposure in $\mathrm{N}_{2}$ is shifted to a lower binding energy, indicating a net reduction of Sn atoms. 

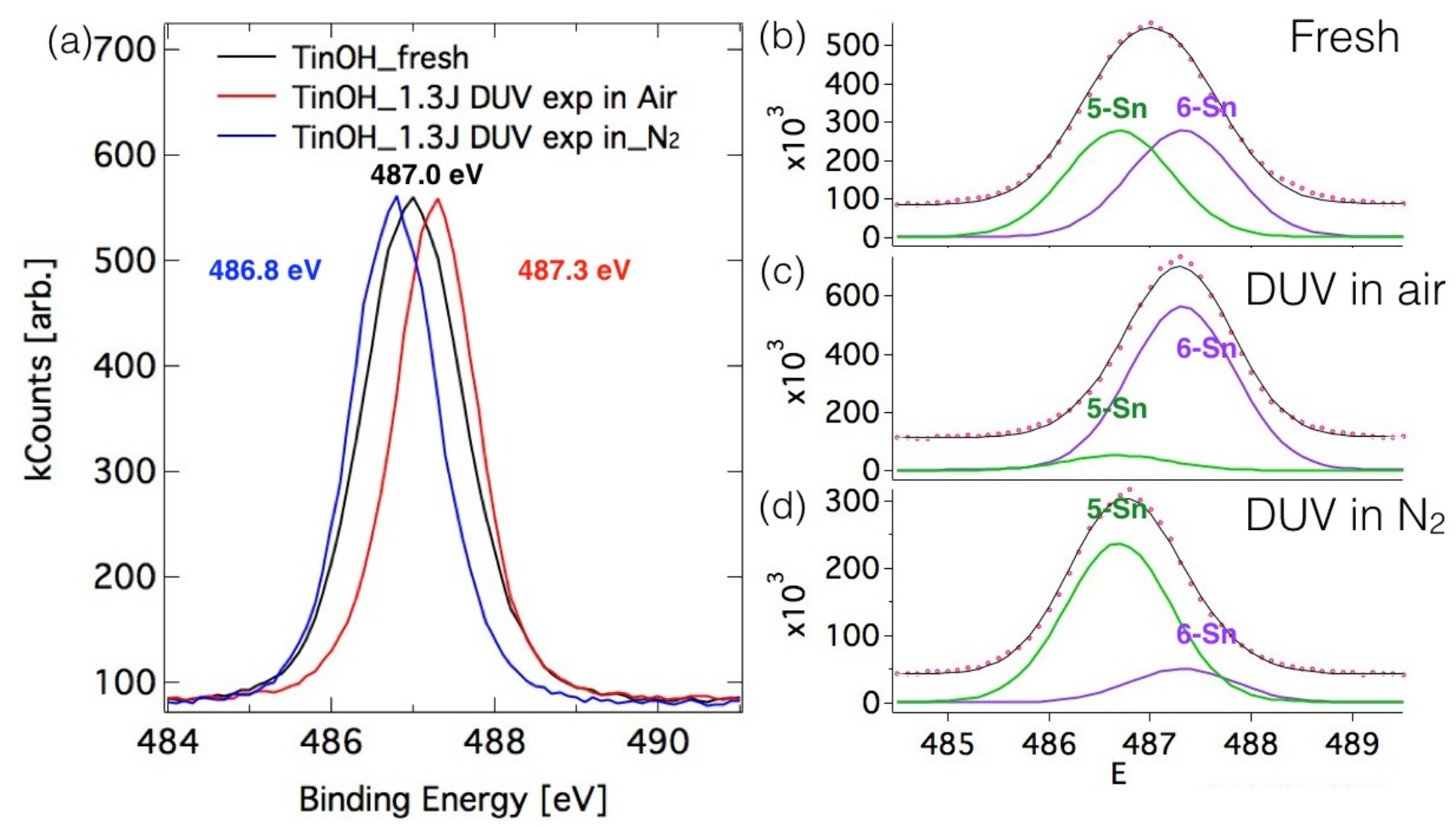

Figure 3) $\mathrm{Sn} 3 \mathrm{~d}_{5 / 2}$ core-level spectra for TinOH fresh sample, TinOH DUV-exposed in air and TinOH DUV-exposed in $\mathrm{N}_{2}$. (a) raw data, (b-d) Fits for each respective sample using the two-component model described in the text.

In panels $3 b-d$ ) least squares fit to the data using two Voigt line-shapes, i.e. each component is a Gaussian convoluted with a Lorentzian function (10\%) that accounts for the finite lifetime of the core ionized state. Because there are two kinds of Sn atoms inside the tin oxo cages (5-coordinated Sn atoms in the center part of molecule, 6-coordinated Sn atoms in the side parts of the molecule facing the counter-ion), two peaks were used to model the spectrum of the fresh sample. Although the photolysis products are probably more complex, we take these peaks to be representative for tin in lower and higher oxidation states, respectively, and use the same peak positions and bandwidths to model the Sn peaks in the exposed samples - leaving only the amplitude as the free parameters. Under these constraints, the spectra in panels 3b-d) were simultaneously fitted for the best global fit describing the full data set.

The results are presented as solid lines in Figs. 3b-d), together with the data as circles. The peak at $486.7 \mathrm{eV}$ (green) corresponds to the 5-coordinated Sn (low oxidation state, low BE) and the peak at $487.3 \mathrm{eV}$ (purple) to the 6-coordinated Sn (higher oxidation state, high BE). In Fig. 3b, the intensity of the two peaks is constrained to $1: 1$ ratio corresponding to the two types of tin. After DUV exposure in air, the component corresponding to the low oxidation state decreases and the intensity is transferred to the peak of the more highly oxidized Sn component. In contrast, the fitting results from the sample exposed in $\mathrm{N}_{2}$ indicated that some of higher oxidation state $\mathrm{Sn}$ was converted to lower oxidation state $\mathrm{Sn}$ atoms. The FWHM of the three spectra were $1.58 \mathrm{eV}, 1.22 \mathrm{eV}$ and $1.27 \mathrm{eV}$, respectively, see panel $3 \mathrm{a}$. The peaks became narrower after exposure because one type of $\mathrm{Sn}$ atom is becoming more dominant - the FWHM values of the underlying components are, however, constant at $1.17 \mathrm{eV}$.

The $\mathrm{O}$ 1s photoelectron spectra are shown in Fig.4. $\mathrm{O}$ 1s peaks from all samples were normalized based on the $\mathrm{O}$ atoms ratio obtained from the overview spectra. Similarly as for the Sn 3d data in figure 3, panel a) shows the O 1s spectra with a common scale on the ordinate axis while panels b-d) show a least squares fit to the data. As seen in panel a), after DUV exposure in air the $\mathrm{O}$ 1s peak shifts to higher binding energy and a considerably higher intensity, while the $\mathrm{O} 1 \mathrm{~s}$ peak of the sample exposed under $\mathrm{N}_{2}$ atmosphere was shifted slightly to lower binding energies while the intensity remains unchanged.[18] 

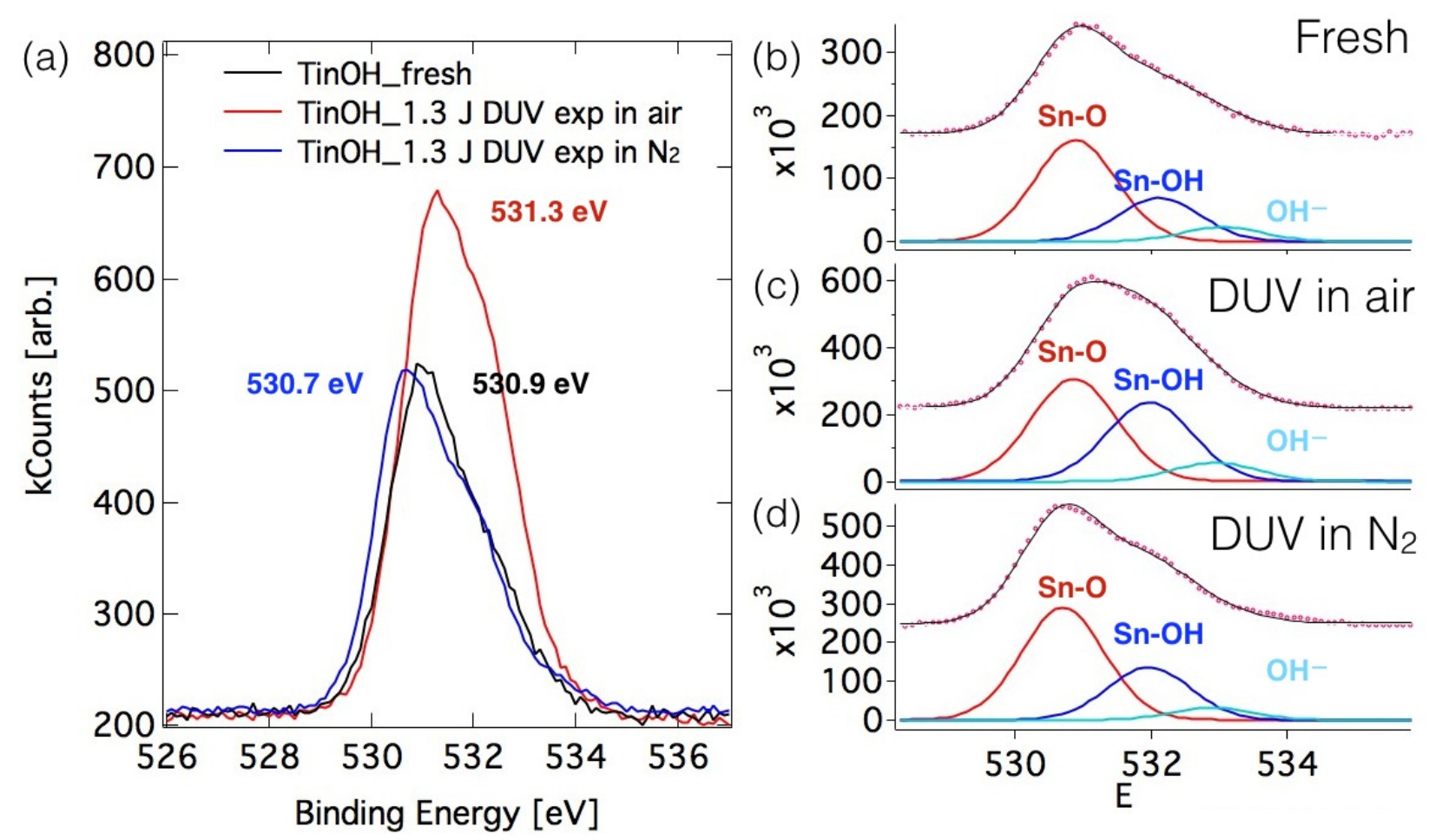

Figure 4. The O1s core level spectra for TinOH fresh sample, sample exposed in air and sample exposed in $\mathrm{N}_{2}$. (a) core level spectra sharing the same scale on the ordinate axis, b-d) least squares fits for each respective sample using the three-component model described in the text.

There are three kinds of $\mathrm{O}$ atoms in the TinOH cage, as can be seen in Fig.1. Three Voigt peaks are assumed in the modelling of the fresh sample, each shown in panel $4 \mathrm{~b}$ ): We assign the low-BE peak (red) to the oxygen atoms bridging three $\mathrm{Sn}$ atoms, the intermediate one (dark blue) to the oxygen in the $\mathrm{OH}$ groups bridging two $\mathrm{Sn}$ atoms and the high-BE (light blue) to the $\mathrm{O}$ in the $\mathrm{OH}^{-}$counterions. In a similar fashion in which we fitted the $\mathrm{Sn} 3 \mathrm{~d}_{5 / 2}$ data, we assume that the underlying spectral features in the spectra of the DUV-exposed samples are the same, i.e. their binding energy positions and linewidth parameters, whereas as the intensities might be altered due to chemical conversion. Under these assumptions the three spectra were fitted simultaneously to get a global optimum fit. The result is shown in Fig. 4b-d).

Table 2. Relative numbers of three types of $\mathrm{O}$ atoms derived from $\mathrm{C} 1 \mathrm{~s}$ spectra as well as extracted binding energies of the respective features (see Fig. 4).

\begin{tabular}{lcccc}
\hline Atom no. & Sn-O & Sn-OH & OH/CO & Total number \\
\hline Binding energy & $530.7 \mathrm{eV}$ & $532.1 \mathrm{eV}$ & $533.0 \mathrm{eV}$ & \\
\hline Expected & 14 & 6 & 2 & 22 \\
TinOH_fresh & 14 & 6 & 2 & 22 \\
DUV exp air & 16 & 13 & 3 & 32 \\
DUV exp N $\mathrm{N}_{2}$ & 14 & 6 & 2 & 22 \\
\hline
\end{tabular}

After the sample has been exposed to DUV in air, all three kinds of $\mathrm{O}$ increased in intensity. Because extensive oxidation has obviously taken place here, most probably resulting in a mixture of oxidation products, the simple approximation with three types of $\mathrm{O}$ is probably not fully valid, but again they do likely represent chemical environments close to those of the fresh material. It is also possible that $\mathrm{O}_{2}^{-}, \mathrm{O}^{-}, \mathrm{O}_{2}{ }^{2-}$ would be chemisorbed on top of the photoresist.[20] For the 
sample exposed to DUV in $\mathrm{N}_{2}$, on the other hand, no oxidation can take place, and the intensity of the three components almost stayed the same. The intensities of the peaks were transferred into the number of oxygen atoms per molecule in the corresponding chemical state; the values are listed in Table 2, alongside with the extracted binding energy of the respective peak.

The C 1s photoelectron spectra were analyzed in the same way as their Sn 3d and O 1s counterparts, as shown in Fig. 5. Panel a) shows the data, normalized by the calculation from the overview spectra of the three samples. There is no clear shift observed in the main low-BE peak of the spectra after exposure, but the intensities and peak shape changes significantly. In the fresh sample, only two kinds of $\mathrm{C}$ atoms inside the tin oxo cage can be distinguished from the spectra, being in Sn-C and C-C bonding configurations. After exposure, however, there is a shoulder observed near $286.1 \mathrm{eV}$ and a new feature around $289.2 \mathrm{eV}$. These features likely arise from $\mathrm{C} 1 \mathrm{~s}$ electron emission from $\mathrm{C}$ atoms with $\mathrm{C}-\mathrm{O}$ and $\mathrm{C}=\mathrm{O}$ bond respectively, as the increased $\mathrm{BE}$ indicates higher oxidation of some of the $\mathrm{C}$ atoms.[22] Thus, four Voigt peaks are used to describe the spectra of the DUV-exposed samples, the results of which are shown in Fig. 5c-d). The extracted concentration of the respective atomic type per molecule and the corresponding binding energies are stated in Table 3. The fresh sample gives the ratio expected from the chemical structure. Furthermore, the $\mathrm{C}$ atom number of atoms in the in C-C and Sn-C configurations from both samples exposed to DUV were decreased. At the same time $\mathrm{C}$ in $\mathrm{C}-\mathrm{O}$ and $\mathrm{C}=\mathrm{O}$ binding motifs were increased slightly, which furthermore demonstrated the oxidation during the DUV exposure. For the sample exposed in air, the $\mathrm{C}-\mathrm{O}$ and $\mathrm{C}=\mathrm{O}$ can be taken as a sign of oxidation on the carbon chain part, in agreement with the increasing of the $\mathrm{O}$ content. But, as we concluded from the overview spectra and $\mathrm{Sn} 3 \mathrm{~d}$, and $\mathrm{O}$ 1s spectra, there was no oxidation or O gain in the sample exposed to DUV in $\mathrm{N}_{2}$. The increase of the $\mathrm{C}=\mathrm{O}$ feature at 289.2 $\mathrm{eV}$ from the sample exposed in $\mathrm{N}_{2}$ must therefore be because of a light-induced rearrangement within the tin-oxo cages. Although there is no extra $\mathrm{O}$ gained, the carbon chain might be cleaved at the $\mathrm{Sn}-\mathrm{C}$ bond or $\mathrm{C}-\mathrm{C}$ bond after which the product could further react with an oxygen atom in the cage to form $\mathrm{C}-\mathrm{O}$ or $\mathrm{C}=\mathrm{O}$ bonds.

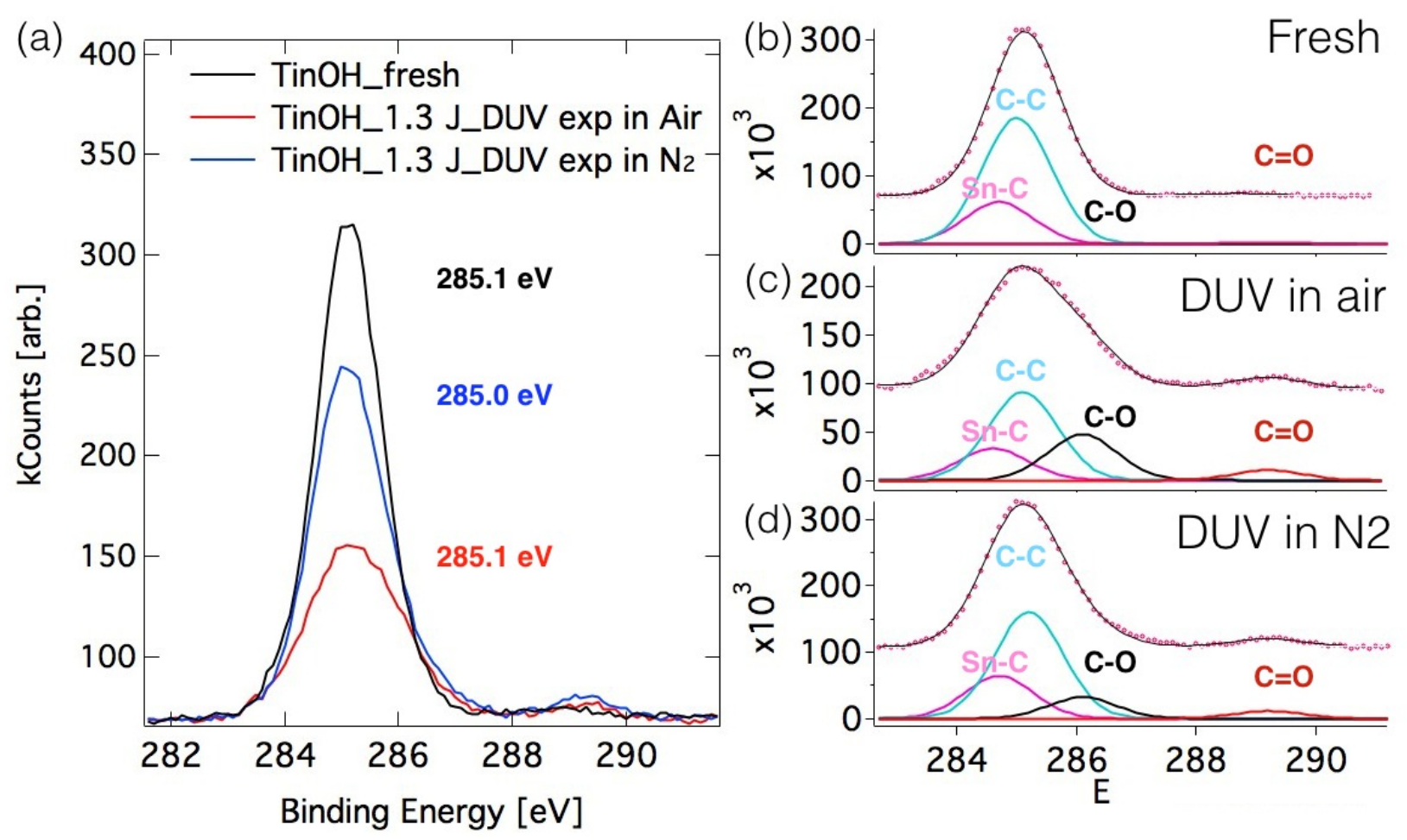

Figure 5) (a) $\mathrm{C}$ 1s core level spectra for TinOH fresh sample, TinOH DUV-exposed in air and TinOH DUV-exposed in $\mathrm{N}_{2}$. Panels (b-d) exhibit fits for each respective sample using the four-component model described in the text. 
Table 3. Relative numbers of four types of $\mathrm{C}$ atoms derived from $\mathrm{C} 1 \mathrm{~s}$ spectra (Fig.5).

\begin{tabular}{lccccc}
\hline Atom no. & Sn-C & C-C & C-O & C=O & Total number \\
\hline Binding energy & $284.9 \mathrm{eV}$ & $285.2 \mathrm{eV}$ & $286.1 \mathrm{eV}$ & $289.2 \mathrm{eV}$ & \\
\hline Expected & 12 & 36 & 0 & 0 & 48 \\
TinOH_fresh & 12 & 36 & 0 & 0 & 48 \\
DUV exp air & 3 & 9 & 5 & 1 & 18 \\
DUV exp N2 & 8 & 22 & 4 & 2 & 36 \\
\hline
\end{tabular}

Based on the analysis of the HAXPES data presented above we propose reaction pathways of the DUV induced reaction, schematically shown in Fig. 6. According to DFT calculations, the Sn-C bond is likely to be initially cleaved under DUV exposure, forming two radicals. If present, oxygen and $\mathrm{H}_{2} \mathrm{O}$ can then directly react with the $\mathrm{Sn}$ radicals and increase the oxidation states of $\mathrm{Sn}$ atoms, which is corresponding to the results shown in Fig. 3 . The butyl radical could further donate a hydrogen atom or abstract one from neighbor cages, to form butane or butene, volatile stable molecules that are readily released into vacuum. A hydrogen atom could be abstracted from a butyl group and form a C-centered radical, which could further react and form side chain oxygenation products that may account for the $289.2 \mathrm{eV}$ band in Fig. 5. For the sample exposed in $\mathrm{N}_{2}$, since there is no active molecule like $\mathrm{O}_{2}$ or $\mathrm{H}_{2} \mathrm{O}$, after the Sn-C bond cleavage occurs, the chance for the radicals to recombine is greater. That would explain why more $\mathrm{C}$ is lost in the sample exposed in air than in the sample exposed in $\mathrm{N}_{2}$. The cleaved butyl radical could also react with the $\mathrm{O}$ inside the cage forming a $\mathrm{C}-\mathrm{O}$ bond, corresponding to the $\mathrm{C}-\mathrm{O}$ peaks in Fig. $5 \mathrm{c}$,d. Another possible reaction is transfer of a hydrogen atom from the butyl radical to the $\mathrm{Sn}$, to form butene and a tin hydride. The formation of such an oxidation-sensitive but otherwise stable reaction product could be the explanation of our finding that the formation of insoluble material after exposure to EUV is enhanced by post-exposure baking.[23]

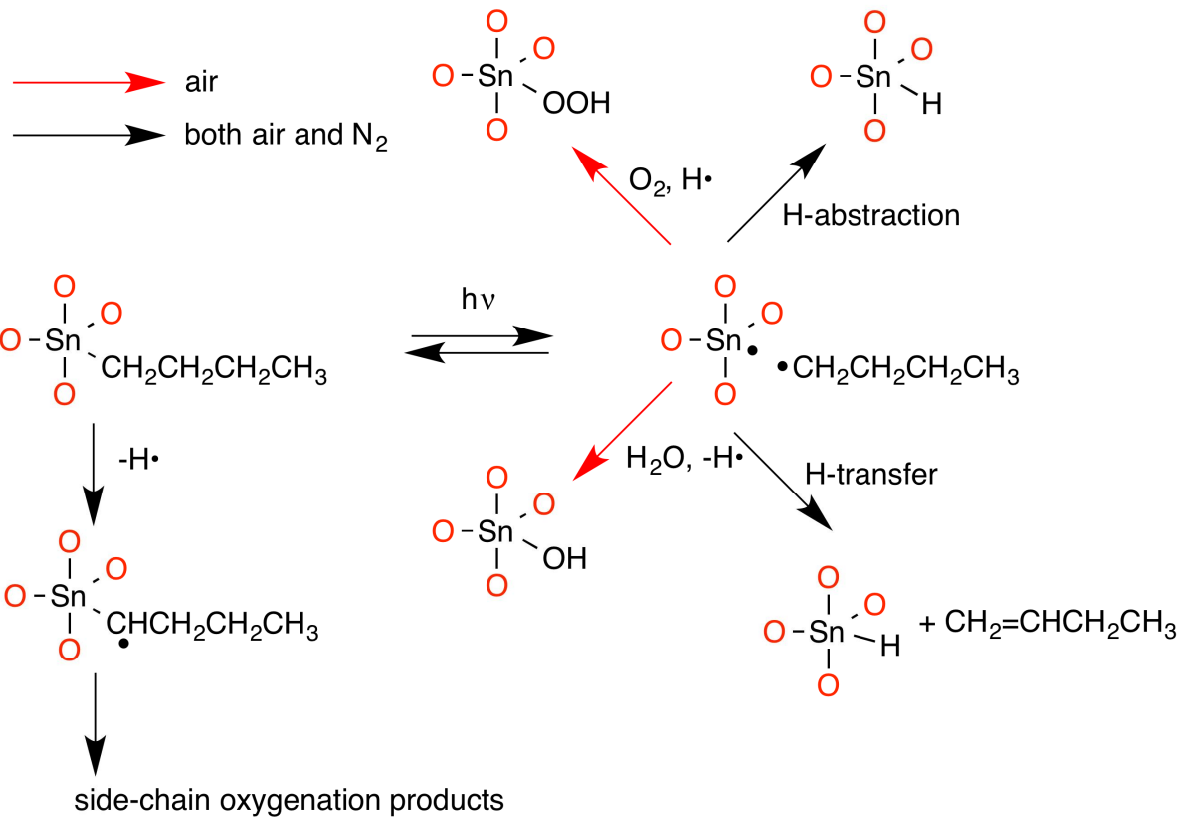

Figure 6) Proposed photochemical mechanism for TinOH exposed by DUV in air and $\mathrm{N}_{2}$. 


\section{CONCLUSION}

HAXPES has been demonstrated to be a powerful tool for analyzing the chemical changes upon DUV exposure in air and under $\mathrm{N}_{2}$ in thin films of tin-oxo cages. Based on the detailed analysis of the presented HAXPES spectra, a mechanism of the photoreactions of TinOH films exposed to DUV in air or under $\mathrm{N}_{2}$ could be proposed.

While very different from the exposure conditions in an EUV lithography tool, the present study highlights the relevance of gas-phase/film interactions for the final photochemical product formation. In ongoing experiments we further compare these results with the changes induced in solution-phase photochemistry of the tin cages, in which products can be isolated and subjected to a wide range of chemical analysis techniques, and with the results of EUV exposures of thin films at $13.5 \mathrm{~nm}$.

\section{ACKNOWLEDGEMENT}

We thank Michiel Hilbers (UvA) and Roberto Felix Duarte (BESSY-II), who provided expertise that greatly assisted the research. We thank HZB for the allocation of neutron/synchrotron radiation beamtime.

\section{REFERENCES}

[1] Li, L., Chakrabarty, S., Spyrou, K., Ober, C.K., Giannelis, E.P., "Studying the Mechanism of Hybrid Nanoparticle Photoresists: Effect of Particle Size on Photopatterning," Chem. Mater., 27, 5027-5031(2015).

[2] Chakrabarty, S., Ouyang, C., Krysak, M., Trikeriotis, M., Cho, K., Giannelis, E.P., Ober, C.K., "Oxide nanoparticle EUV resists: toward understanding the mechanism of positive and negative tone patterning," Proc. SPIE 8679, 1-8 (2013).

[3] Chakrabarty, S., Sarma, C., Li, L., Giannelis, E.P., Ober, C.K., "Increasing sensitivity of oxide nanoparticle photoresists," Proc. SPIE 9048, 1C-1-5(2014).

[4] Stowers, J., Keszler, D.A., "High resolution, high sensitivity inorganic resists," Microelectron. Eng., 86, 730733(2009).

[5] Meyers, T.S. Anderson, T.J., Edson, B.J., Jiang, K., Keszler, A.D., Kocsis, K.M., Telecky, J.A., Cardineau, J.B., "Organometallic solution based high resolution patterning compositions and corresponding methods," US 2016/0116839 A1, (2015).

[6] Cardineau, B., Del Re, R., Marnell, M., Al-Mashat, H., Vockenhuber, M., Ekinci, Y., Sarma, C., Freedman, D.A., Brainard, R.L., "Photolithographic properties of tin-oxo clusters using extreme ultraviolet light (13.5nm)," Microelectron. Eng.127, 44-50(2014).

[7] Sortland, M., Del Re, R., Passarelli, J., Hotalen, J., Vockenhuber, M., Ekinci, Y., Neisser, M., Freedmaan, D., Brainard, R.L., "Positive-tone EUV resists: complexes of platinum and palladium," Proc. SPIE 942227, 1-9(2015).

[8] Del Re, R., Sortland, M., Pasarelli, J., Cardineau, B., Ekinci, Y., Vockenhuber, M., Neisser, M., Freedmaan, D., Brainard, R.L.,"Low-LER tin carboxylate photoresists using EUV," Proc. SPIE 942221, 1-10(2015).

[9] Hassanein, E., Higgins, C., Naulleau, P., Matyi, R., Gallatin, G., Denbeaux, G., Antohe, A., Thackeray, J., Spear, K., Szmanda, C., Anderson, C.N., Niakoula, D., Malloy, M., Khurshid, A., Montgomery, C., Piscani, E.C., Rudack, A., Byers, J., Ma, A., Dean, K., Brainard, R., "Film quantum yields of ultrahigh PAG EUV photoresists," Proc. SPIE 69211, 13(2008).

[10] Kozawa, T., "Lower Limit of Line Edge Roughness in High-Dose Exposure of Chemically Amplified Extreme Ultraviolet Resists," Jpn. J. Appl. Phys., 51, 06FC1(2012).

[11] Fondell, M., Gorgoi, M., Boman, M., Lindblad, A., "An HAXPES study of Sn, SnS, SnO and $\mathrm{SnO}_{2}$," J. Electron. Spectrosc. Relat. Phenom., 195, 195-199(2014).

[12] Banse, F., Ribot, F., Toladeno, P., Maquet, J., Sanchez, C., "Hydrolysis of monobutyltin Trialkoxides synthesis and characterizations of $\left\{(\mathrm{BuSn})_{12} \mathrm{O}_{14}(\mathrm{OH})_{6}\right\}(\mathrm{OH})_{2}$," Inorg. Chem., 34, 9(1995). 
[13] Henke, B.L., Gullikson, E.M., Davis, J.C., "X-Ray Interactions: Photoabsorption, Scattering, Transmission, and Reflection at $\mathrm{E}=50-30,000 \mathrm{eV}, \mathrm{Z}=1-92$. Atomic Data and Nuclear Data Tables,", 54, 181-342(1993).

[14] Fallica, R., Stowers, J.K., Grenville, A., Frommhold, A., Robinson, A.P.G., Ekinci, Y., "Dynamic absorption coefficients of chemically amplified resists and nonchemically amplified resists at extreme ultraviolet," J.

Micro/Nanolith. MEMS MOEMS,15, 033506, 1-7(2016).

[15] Mårtensson, N., Sokolowski, E., Svensson, S., "50 years anniversary of the discovery of the core level chemical shifts. The early years of photoelectron spectroscopy,” J. Electron. Spectrosc. Relat. Phenom.,193, 27-33(2014).

[16] Schaefers, F., Mertin, M., Gorgoi, M., et al. "KMC-1: A high resolution and high flux soft x-ray beamline at BESSY,” Rev. Sci. Instrum. 78, 123102(2007).

[17] Seah, M.P., "Post-1989 calibration energies for X-ray photoelectron spectrometers and the 1990 Josephson constant," Surf. Interface Anal., 14, 488-488(1989).

[18] Xiao, X., Han, B., Deng, D., Cai, X., and Wang, Y., "Enhanced formaldehyde sensing properties of $\mathrm{SnO}_{2}$ nanorods coupled with $\mathrm{Zn}_{2} \mathrm{SnO}_{4}$," RSC Advances, 5, 42628-42636(2015).

[19] Liu, C., Huang, H., Cao, G., Xue, F., Paredes Camacho R.A., Dong X., "Enhanced Electrochemical Stability of SnCarbon Nanotube Nanocapsules as Lithium-Ion Battery Anode," Electrochim. Acta,144, 376-382(2014).

[20] Kawabe, T., Tabata, K., Suzuki, E., Yamaguchi, Y., Nagasawa, Y., "Electronic States of Chemisorbed Oxygen Species and Their Mutually Related Studies on $\mathrm{SnO}_{2}$ Thin Film,” J. Phys. Chem. B, 105, 4239-4244(2011).

[21] Trzhaskovskaya, M.B., Nefedov V.I., Yarzhemsky V.G., "Photoelectron Angular distribution parameters for Elements $Z=1$ to $Z=54$ in the photoelectron energy range 100-5000 eV," Atomic Data and Nuclear Data Tables, 77, 97$159(2001)$.

[22] Preuss, C.M., Tischer, T., Rodriguez-Emmenegger, C., Zieger, M.M, Bruns, M., Goldmann, A.S., Barner-Kowollik, C., "A bioinspired light induced avenue for the design of patterned functional interfaces," J. Mater. Chem. B, 2, 3640(2014).

[23] Haitjema, J., Zhang, Y., Vockenhuber, M., Kazazis, D., Ekinci, Y., Brouwer, A.M., "Extreme ultraviolet patterning of tin-oxo cages," Proc. SPIE 10143, 72(2017). 